\title{
Altos estructurales costa afuera en la región de Valparaíso, Chile central: Réplica a comentarios de C. Rodrigo
}

\author{
Offshore structural highs at Valparaíso region, central Chile: Reply to C. Rodrigo’́s statements
}

\section{Hernán P. Vergara ${ }^{1}$ y René Astudillo ${ }^{1}$}

\begin{abstract}
${ }^{1}$ Facultad de Ciencias del Mar y de Recursos Naturales, Universidad de Valparaíso, Casilla 5080 Reñaca, Viña del Mar, Chile. hernan.vergara@uv.cl

Abstract.- C. Rodrigo states that the satellite altimetry data used by the authors is no longer valid and, therefore, the 3 summits are non-existent. The author's opinion is that the version compiled from Sandwell \& Smith (2001), showing 3 summits, as much as the one from Rodrigo (2011), showing only one, deserves respect. Both must be considered as proposals and should be judged by the scientific community for their approval or rejection.
\end{abstract}

Key words: Data bank, satellite altimetry, non-confined block, slump, slope

\section{INTRODUCCIÓN}

La Nota Científica publicada por Rodrigo 2011 (en adelante CR), concluye que las 3 cumbres submarinas orientadas $\mathrm{N} 10^{\circ} \mathrm{W}$, no existen, porque en su opinión, la cartografía mediante altimetría satelital utilizada para inferirlas (Sandwell \& Smith 2001), es inexacta. Por lo anterior, se hace necesario esclarecer y dar respuesta con mención a factores batimétricos y geológicos del área del margen continental de la Región de Valparaíso comprendida entre Papudo y Quintero.

\section{RESPUESTA A LOS COMENTARIOS}

Se agradece el aporte, por cuanto dicha área no ha sido suficientemente estudiada, así como la re-interpretación cartográfica, donde se establece la precedencia de la toponimia ‘Cañón Aconcagua' sobre 'Cañón Montemar', por lo cual en la Figura 1 se ha incluido la nueva denominación (Fig 1b). Su información de batimetría multihaz del AGOR Vidal Gormaz y del RV Sonne, en términos generales, enriquecen la morfología submarina del área, considerando que la primera información respecto del área de estudio relacionada con las geociencias marinas corresponde a Scholl et al. (1970) al interpretar un perfil sísmico obtenido en 1967 durante el crucero del USN Charles Davis, siendo la primera vez que se tuvo noticia del grado de deformación del talud inferior (actualmente interpretado como un talud acrecional) y del talud medio (interpretado entonces como una terraza tectónica y hoy conocida como la Cuenca Valparaíso) durante el crucero del RV Sonne de 1995 efectuado como parte del proyecto CONDOR [Chilean Offshore Natural Disaster and Ocean-Environmental Research] (Von Huene et al. 1997).

No obstante el aporte batimétrico y toponímico efectuado por CR, sus comentarios contienen imprecisiones, omisiones y errores que se detallan a continuación:

a) En la Introducción, párrafo 2, líneas 12-13, CR dice «... que produjeron un levantamiento de un bloque no confinado del borde externo de la plataforma».

$\mathrm{Al}$ respecto la réplica es: en el artículo no se menciona que los altos topográficos se levantaron. Por el contrario, el bloque no confinado fue dislocado y por ello todo el lineamiento se deslizó gravitacionalmente por el talud provocando la profundización (subsidencia) del referido sector y no su elevación como afirma CR (Resultados y Discusión, párrafo 3).

b) En la Introducción, párrafo 2, línea 9, CR dice: «Sostienen que su origen pueda estar relacionado con procesos tectónicos...»

La palabra sostienen, es una creación de CR, quien se permite modificar el texto y atribuir consecuencias que no son aplicables al caso. En realidad los autores expresaron: «El aspecto de la morfología generalizada... sugiere el desplazamiento vertical de un bloque no confinado del borde externo de la plataforma» (Resultados y Discusión, párrafo 3). Se estima que 'sostener' y 'sugerir' no son sinónimos, por cuanto el primer término implica 

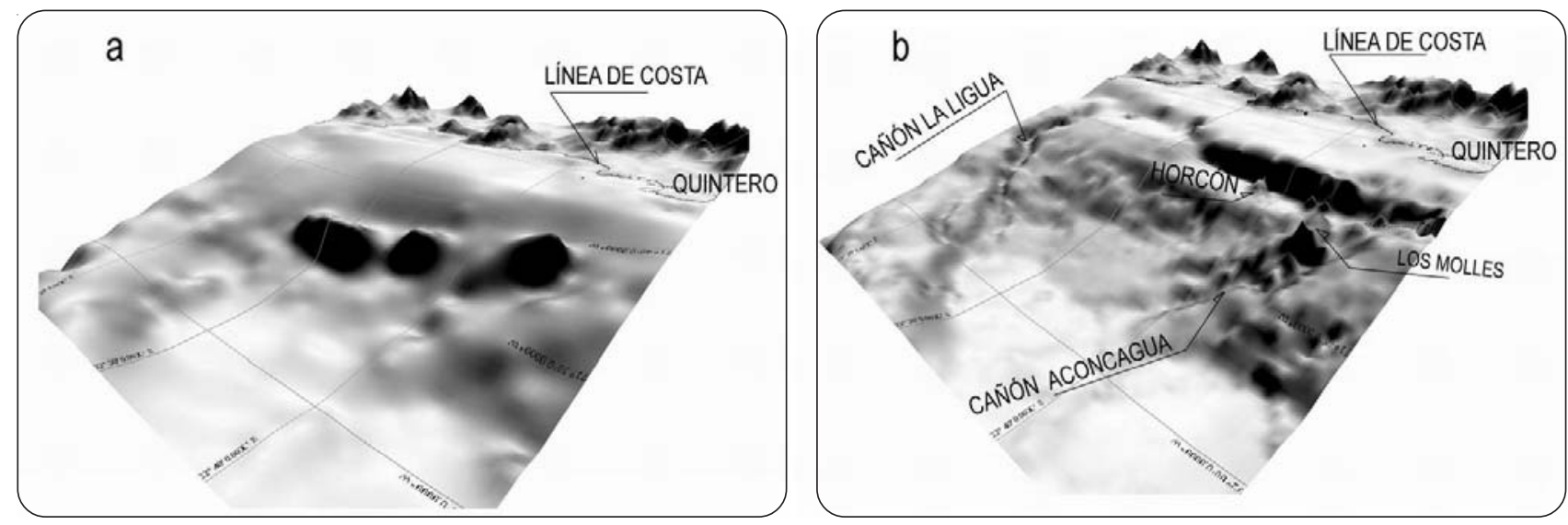

Figura 1. a) Bloque 3D basado en los datos de Sandwell \& Smith (2001), que ilustra la ubicación de las 3 cumbres descritas en Vergara \& Astudillo (2008); b) ilustra la misma área, pero basada en Satellite Geodesy (UCSD) ${ }^{3}$, con solamente 2 cumbres / a) 3D block based on Sandwell \& Smith (2001), showing the 3 summits described in Vergara \& Astudillo (2008); b) shows the same area but according to the Satellite Geodesy $(\mathrm{UCSD})^{3}$, showing just two summits

una afirmación tajante y el segundo conlleva implícitamente una incertidumbre.

c) En Introducción, párrafo 3, líneas 7-8 y 12-14 CR dice: «... e influenciados por la subducción de la dorsal o cadena de Juan Fernández» y «la cual es inducida por la subducción de montes marinos y cordones de la dorsal de Juan Fernández».

Considerando que el crucero Sonne realizado entre marzo y julio de 1995 corroboró que el archipiélago Juan Fernández está complementado en forma subacuática por una alineación de aparatos volcánicos submarinos tanto al Este como al Oeste de las 3 islas (Vergara \& Morales 1985) y es evidente que la discontinuidad implica la inexistencia de una dorsal como lo menciona Rodrigo 2011. Consecuentemente, no hay claridad conceptual al denominar al mismo rasgo morfológico indistintamente como dorsal, cadena, cordón y montes submarinos, ya que genéticamente ellos tienen orígenes distintos (OHICOI 2010). Por esta razón, tanto en el título como en el texto del artículo, la expresión altos estructurales se usó para referirse a cumbres que genéticamente difieren de los montes, dorsales, cordones y cadenas submarinas.

d) En Materiales y métodos, párrafo 1, líneas 1-8, CR dice: «La base de datos utilizada por Vergara \& Astudillo (2008) consiste de datos topográficos terrestres obtenidos por la SRTM y datos de topografía submarina que incluye la batimetría derivada de altimetría satelital
(Smith \& Sandwell 1997) y la obtenida por sondajes de buques de las siguientes instituciones principales: Lamont-Doherty Earth Observatory (Columbia University), National Geophysical Data Center (NGDC) y SIO, entre otras.»

Nuevamente CR incurre en un error y a la vez modificó el texto del artículo. En primer lugar, la base de datos que los autores emplearon corresponde a Sandwell \& Smith 2001 y no 1997. En cuanto a lo segundo, en Materiales y métodos los autores no mencionan fuentes batimétricas basadas en sondajes de buques tales como LamontDoherty Earth Observatory (Columbia University), National Geophysical Data Center (NGDC) y SIO. La única base de datos utilizada es de altimetría satelital y está señalado también en el Resumen del artículo. Efectuar modificaciones en textos de otros especialistas constituye una costumbre inusual que es aconsejable sea corregida.

e) En Resultados y discusión, párrafos 5 (líneas 19-23) y 6 (líneas 14-18) CR dice: «El 'track' removido correspondió al ODP141JR, crucero perteneciente al programa Ocean Drilling Program Leg 141, cuyos datos batimétricos fueron obtenidos a bordo del buque $R / V$ 'Joides Resolution' en 1992» y "Se comprueba e identifica que la causa de la aparición de los altos estructurales..., es la incorporación de datos batimétricos erróneos de tipo monohaz de un sondaje específico efectuado por el buque $R / V$ 'Joides Resolution'». 
Al respecto, cabe precisar que esa afirmación no es verídica porque la nave JOIDES ${ }^{1}$ Resolution no realizaba batimetría. Las 3 naves que han sido plataformas de los programas D-SDP (Glomar Challenger: 1968-1985), ODP (JOIDES Resolution: 1985-2003) e IODP (Chikyu: 2003 a la fecha), su única actividad ha sido perforar el fondo marino de modo similar al de la actividad petrolera. Como base de trabajo usan información previa de varios cruceros 'tradicionales' y en base a eso se definen objetivos, se seleccionan las áreas de interés, se define el número de pozos y los sitios exactos a perforar. Entre el 12 de noviembre de 1991 y el 12 de enero de 1992 empleando el JOIDES Resolution se perforaron 5 sitios y 13 pozos en el talud continental frente a la Región de Aysén, como parte del programa internacional Ocean Drilling Program (ODP), crucero 141, en un área donde la dorsal de Chile intersecta Sudamérica.

Además - y no por ello menos importante- uno de los autores del artículo participó en el crucero ODP $141^{2}$. En dicha referencia pueden comprobarse los objetivos del crucero, donde se constata que no realizó tareas batimétricas y que tanto el zarpe hacia el área de trabajo como la recalada fueron en Valparaíso en las fechas mencionadas. Además, la actividad batimétrica nunca fue incluida en el permiso otorgado por el gobierno chileno para trabajar en sus aguas jurisdiccionales. Finalmente y como un dato marginal, debe consignarse que la designación oficial del JOIDES Resolution fue DV (Drilling Vessel) y no RV (Research Vessel) como asevera CR.

f) En Resultados y discusión, párrafo 3, líneas 5-8 CR dice: «Estos canales constituyen depresiones alargadas que posiblemente conduzcan sedimentos desde la plataforma hacia mayores profundidades».

Se consigna que la existencia de canales o surcos visibles en las reconstrucciones que efectúa CR puede explicarse por lineamientos estructurales (fallas) o por erosión entre otras posibilidades. Ellos se visualizan con claridad en Vergara (1996), figura 5 y Vergara (1998), figura 2.

g) La credibilidad de los nuevos gráficos aportados por CR también son susceptibles de críticas debido a que la batimetría que aporta respecto al JOIDES Resolution es inexistente.

h) CR incluye en su base de datos la versión SRTM Plus 2008, actualizada a noviembre de 2009 (Becker et al. 2009) y parece asumir que tanto la base de datos utilizada como la fecha de la publicación corresponden al mismo año (2008). Sin embargo, su versión fue actualizada posteriormente respecto a la de los autores (Rodrigo 2011). Mas tarde en diciembre de 2011 (Satellite Geodesy ${ }^{3}$ ) fue puesta a disposición de la comunidad científica una nueva actualización, en que se emplearon nuevos algoritmos matemáticos que han suavizado rasgos como las cumbres y depresiones del relieve submarino, lo que ha ocasionado un cierto 'aplanamiento' de algunos rasgos del relieve subacuático. Basados en esta última versión, los autores incluyen la Fig. 1a, correspondiente al artículo Vergara \& Astudillo 2008, que se compara con la nueva versión de Sandwell \& Smith (2011) (Fig. 1b) donde se puede apreciar que la cumbre Los Molles ('C' de CR) continua apareciendo, la cumbre Horcón ('B' de CR) está suavizada y desplazada levemente al este,y la cumbre Zapallar ('A' de CR) ya no aparece.

\section{Conclusiones}

a) Lo positivo de la propuesta de CR se relaciona con la batimetría del AGOR Vidal Gormaz y el RV Sonne, especialmente la primera y que, lamentablemente, por haber sido publicada institucionalmente, con reducida divulgación, y no en una revista científica, no estuvo sino hasta ahora en conocimiento de los autores. Ambas bases de datos contribuyen a incrementar el conocimiento en cuanto a la morfología submarina, siendo posible inferir aspectos tectónicos, donde históricamente se halla una nucleación sísmica importante. Los autores estiman que ambos aportes contribuyen con este paso inicial (objetivo del artículo de Vergara \& Astudillo 2008) y sería deseable se complementase con la necesaria información geofísica que permita aclarar esa problemática.

b) De la lectura de la Nota Científica se advierten errores de carácter conceptual por parte de CR respecto a la

\footnotetext{
${ }^{1}$ JOIDES = Joint Institutions for Deep Earth Sampling

${ }^{2}<$ http://www-odp.tamu.edu/publications/prelim/141PREL.PDF>

${ }^{3}$ Satellite Geodesy, University of California San Diego, Scripps Institution of Oceanography, La Jolla <ftp://topex.ucsd.edu/pub/ srtm30_plus/-SRTM30_PLUS_V8.-kmz>
} 
morfología y al tectonismo del área de interés, que lamentablemente inducen a cometer algunas imprecisiones. Se suma la inclusión de una base de datos que no corresponde a la nave que según él los habría obtenido, por lo tanto se sugiere prolijidad en la entrega de información, y CR deberá verificar la veracidad de sus comentarios para que las dudas conceptuales sean aclarada, en beneficio de la credibilidad científica.

c) De los datos expuestos aquí los autores infieren que ambas partes carecen de evidencias suficientes y objetivas para concluir la calidad de la información del relieve submarino del área. Ambas versiones deben considerarse como proposiciones y su aceptación o rechazo corresponde a la comunidad científica. Asimismo, los autores consideran que lo anterior no invalida la información ni la metodología empleada en el artículo.

d) Una forma de clarificar fehacientemente la situación es la programación de un crucero en el área en cuestión por parte de una nave con sistema multihaz. Tal tecnología es factible utilizar a través del buque oceanográfico 'Cabo de Hornos’, a disposición de la comunidad científica marina y pesquera. La postulación y adjudicación a proyectos concursables permitiría desarrollar dicha tarea.

\section{LITERATURA CITADA}

Becker JJ, DT Sandwell, WHF Smith, J Braud, B Binder, J Depner, D Fabre, J Factor, S Ingalls, S-H Kim, R Ladner, K Marks, S Nelson, A Pharaoh, S Sharman, R Trimmer, J von Rosenberg, G Wallace \& P Weatherall. 2009. Global bathymetry and elevation data at 30 arc seconds resolution: SRTM30 Plus. Marine Geodesy 32(4): 355-371.
OHI-COI. 2010. GEBCO Gazetteer of Undersea Feature Names. OHI-COI Publication B-8, 409 pp. International Hydrographic Bureau, Monaco.

Rodrigo C. 2011. Interpretación batimétrica del área de Valparaíso: comentarios sobre el artículo de HP Vergara \& R Astudillo, 'Altos estructurales costa afuera en la región de Valparaíso, Chile central’. Revista de Biología Marina y Oceanografía 46(2): 249-255.

Sandwell DT \& WHF Smith. 2001. Bathymetric estimation. In: Fu L-L \& A Cazenave (eds). Satellite altimetry and earth sciences. International Geophysical Series 69: 441-457, Academic Press, New York.

Scholl DW, MN Christensen, R von Huene \& M Marlow. 1970. Peru-Chile trench sediments and sea-floor spreading. Geological Society of America Bulletin 81: 1339-1360.

Vergara H. 1996. La Cuenca de Valparaíso: antecedentes morfotectónicos y sedimentológicos. Revista de Biología Marina 31(1): 45-63.

Vergara H. 1998. Efectos morfotectónicos en el margen chileno $\left(32^{\circ}-34^{\circ} \mathrm{S}\right)$ debido a la subducción del cordón asísmico Juan Fernández. Revista de Biología Marina y Oceanografía 33(2): 199-212.

Vergara H \& E Morales. 1985. Morfología submarina del segmento central del cordón asísmico Juan Fernández, Pacífico Suroriental. En: Arana P (ed). Investigaciones Marinas en el Archipiélago de Juan Fernández, pp. 25-34. Escuela de Ciencias del Mar, Universidad Católica de Valparaíso, Valparaíso.

Vergara H \& R Astudillo. 2008. Altos estructurales costa afuera en la región de Valparaíso, Chile central. Revista de Biología Marina y Oceanografía 43(3): 539-545

Von Huene R, J Corvalán, ER Flueh, K Hinz, J Korstgard, CR Ranero, W Weinrebe \& the CONDOR Scientist. 1997. Tectonic control of the subducting Juan Fernández Ridge on the Andean margin near Valparaiso, Chile. Tectonics 16(3): 474-488. 